\title{
Theory of Communotion and the Evolution of Chinese Syntactic Properties
}

\author{
Zhongxin Dai \\ School of Foreign Languages, North China Electric Power University \\ Beijing 102206, China
}

\begin{abstract}
The unique syntactic properties of the Chinese language have been universally discussed among Chinese linguists, but the genotypic source of the properties has rarely been explored. This article attempted to explore the source from human communicative notion (communotion). Communotion is the intended notion emerging in the interlocutor's mind in the context of communication, and impelling him to struggle to express with whatever tool is available. The syntactic properties of human particular languages have evolved from the immanent relationships between the elements in the communotion. This article identifies five design principles underlying the heterogeneous transduction from the hierarchical notional relationships in the communotion to the hierarchical syntactic structures in the Chinese language: Situatedness, Theme Depiction, Dynamic Focalization, Phrase-based Construction and Word Order. The incongruity between the syntactic properties of the Chinese language and other languages is caused by the diversity of the sociocultural conventional verbalization in the realization of the expression of the communotion.
\end{abstract}

Keywords: communotion, communication, notion, evolution, Chinese language, syntactic property

\section{INTRODUCTION}

It is well known that the syntactic structures of the Chinese language are characterized by such unique properties as topic-prominence, run-on syntactic structures, four-character idiomatic expressions and flexible word order. But it is less known that these observable phenotypic syntactic properties have evolved from the underlying genotypic message instructions of the communicative notion (henceforth blended as communotion) of the Chinese people over at least thousands of years.

In the early 1980s, many Chinese linguists (e.g. Wangdao Chen, Shaoyu Guo, Shilu Zhang and Zhigong Zhang, see the review on cultural linguistics in China by Shen [1]) questioned the legitimacy and adequacy of the grammar of the Chinese language, which had been investigated and formulated by modern Chinese linguists, emulating European linguists in their description of languages such as English. The conception of grammar was introduced to Chinese scholars more than a century ago by Jianzhong Ma [2] in his publication of the first Chinese grammar. For thousands of years, the study of the Chinese language has focused on words or such structures as syntactic parallels and contrasts. The domains of language grammar that Western grammarians have been concerned about have historically never become salient issues. Those modern Chinese linguists reviewed by Shen [1] argued that Western theories in general linguistics had not taken into account the facts and laws of the Chinese language, and, therefore, did not apply to Chinese. They thought that grammarians in China had overly followed the Western theoretical framework, intentionally accommodated to the Western theoretical parameters, and not taken into account the basic properties of the Chinese language. They contended that theories should always come from practice, and that theories on the grammar of the Chinese language should have been based on the investigation of the 
Chinese language. For instance, the Chinese language is typically characterized by 'flowing sentences' $[3,4]$, but this quintessential property of the Chinese language cannot find its niche in the current syntactic theories in Western linguistics.

The struggle of the Chinese linguists to shake off the shackles of the Western syntactic theories has not resulted in a systematic syntactic theory for the Chinese language. Increasing evidence has been uncovered which indicates that the Chinese language seems to belong to another totally different branch of human language, since it cannot be adequately and topologically explained within the fundamental syntactic theories for Western languages such as English.

This is the thorny and vexed issue that Chinese linguists will have to deal with. On the one hand, the conception of the universality of human language requires the Chinese language and some other similar East Asian languages to be legitimately and typologically interpreted as another main branch of human language that shares the same origin with other branches of human languages. On the other, the uniqueness of Chinese syntax needs systematic genotypic explanation as to the underlying design principles that have shaped Chinese as it is. Inquiry into the underlying motivation in the evolutionary process of the shaping and development of the Chinese language must go beyond the practice of comparison of the Chinese language with other languages such as English. Comparison between languages can generate some descriptive data for the differences between them, but cannot account for the genotypic motivation for the phenotype of the Chinese syntax.

Justification for the syntactic uniqueness of the Chinese language requires close examination of the communotion source where human language originated. Since as yet there has been no general agreement among scholars interested in human language evolution, I have to explore the issue on my own by tracing Chinese and English, the two languages that I have learned, to the point where the verbalized languages reveal, in language communication, their Being (the concept of Being is derived from Heidegger [5]) that can be both housed in Chinese and English (the core theory in language translation and interpretation), and by analyzing the phylogenetic and ontogenetic sources of communotion that has impelled humans to create their language and children in an established community to learn the language of the community.

This approach to the issue is intended to unveil the Being and the shaping of the Being of human language, and by doing so I shall be in a position to delineate the origin, shaping and development of the phenotype of the Chinese language from the genotype of the communotion of the ancestors of the Chinese people. Firstly, I shall formulate the theory of human communotion and discuss the nature of the communotion by elaborating on the something that "the men in the making" "had to say to each other" in Engels' assertion about the evolution of human language[6], by looking at the thought that Vygotsky [7] meant when he was discussing the speaking process, and by reviewing Bloomfield's [8] observation of the first word $d a$ produced by an infant. Secondly, based upon the theory of communotion, I shall address the issue of the syntactic uniqueness of the Chinese language by illuminating the transduction of the genotype of the communotion into the phenotypic syntactic properties of the Chinese language, and at the same time identify the design principles underpinning the transduction. And thirdly, I shall account for some basic properties of the Chinese language on the basis of these underpinning design principles.

\section{THEORY OF HUMAN COMMUNOTION}

Human communotion refers to the mental and psychological entity that is brought into being by the communicative intention of humans in real or imagined communicative situations. It is a 
continuation of nonhuman communotion of our simian ancestors, and similar to that of other animals which are capable of communicating their needs.

Human beings evolved from their simian ancestors under the pressure of their living environment. In order to adapt to the environment, our ancestors had to labor. According to Engels [6], labor, in the proper sense of the word, began with making tools. Labor made "the characteristic difference between the troupe of monkeys and human society." With the development of labor and tools, human society came into play, which is different from the troupe of apes. It was labor that made our gregarious ancestors even more gregarious.

[T] he development of labour necessarily helped to bring the members of society closer together by increasing cases of mutual support and joint activity, and by making clear the advantage of this joint activity to each individual. In short, men in the making arrived at the point where they had something to say to each other [6].

The italicized part in the sentence was emphasized by Engels himself. Why did he stress this part? It might be that he thought it was the very stuff that had initiated the evolution of human language.

Engels asserted that "[c]omparison with animals proves that this explanation of the origin of language from and in the process of labour is the only correct one." Animals did not develop their language because they did not form the kind of society based upon labor and hence they had very little to communicate. "The little that even the most highly-developed animals need to communicate to each other does not require articulate speech." Nevertheless, the tamed dog and the tamed horse "have acquired the capacity for feelings such as affection for man, gratitude, etc.," and "in many cases they now feel their inability to speak as a defect, ...."

Animals do not labor, and therefore they have not developed the capability to make physical tools to solve problems encountered in labor. Since they cannot frequently and routinely resort to a third party to solve the simplest problems in labor, it would be infinitely impossible for them to develop an intangible tool to solve the sociocultural, psychological and mental problem of communication. Any investigation into the evolution of human language by examination of animals will turn out to be a waste of effort, time and talent.

The concept of communotion is different from the concept of "communicative intention" in pragmatics or in the study of human language evolution from the perspective of pragmatics theories (e.g. Scott-Phillips [9]). Pragmatics studies the artistic use of a mature human language in human linguistic communication where the focus is on the comprehension of language in use. Communotion is mainly concerned with the primordial expression of the something in Engels' statement. It is somewhat like the definition of language that Sapir [10: 8] spelled out about a century ago: the "ideas, emotions, and desires" to be communicated "by means of a system of voluntarily produced symbols." He said that "[l]anguage is a purely human and non-instinctive method" for communication. "There is no discernible instinctive basis in human speech." This suggests that human language has evolved as a symbolic tool intended to express the communotion.

In the following, I shall try to elucidate what the communotion (or the something in Engels' discussion) was or looked like in the mind of the men in the making. We shall have to imagine the scenarios of our gregarious and sociable ancestors, who had been impelled by the internal communotion (the selective pressure) to resort to a third party (a tool of human language) to indicate to the targeted audience what it was in the mind that they desperately needed to say to them. 
This something in the mind of our ancestors bears comparison with that in the mind of toddlers and with that in the mind of every one of us when we attempt to communicate something to each other in our native or foreign language. It is this mental and psychological entity that I am at this very moment definitely feeling and desperately struggling to express. Of course, there is little doubt that the communotion of the something in our mind when we are communicating with people in the same community or with foreigners differs from that of our ancestors in that our communotion has been partially verbalized by our native language and, sometimes, by other languages we have learned. But if we want to convey our communotion to the foreigners, we would have to de-verbalize the communotion to the original something in order to express it in the conventional fashion of the intended target foreign language. The very fact that human languages are translatable and interpretable to one another suggests that there is something behind or beyond the phenotypic properties of human particular languages. If there exists anything universal in human languages, it is the content of the communotion that is universal.

Then what is the communotion that I am struggling to get out of my mind, and how does it come into being? In order to have a clear conception of the communotion, we shall have to introduce Heidegger's [5] phenomenal and existential conception of the 'Dasein' (the literal German meaning is being there or presence). The term denotes the way of human beings being engaged in the world with other beings. The capitalized Being refers to the essence of beings, different from a being or beings, which are observable and touchable objects in the outside world. The Being of human beings differs from the Being of other beings in that it is endowed with the capability to comprehend, interpret and care for the Being of other beings. But the exercise of the comprehension and interpretation is conditional upon the involvement of the Dasein within the world with other beings functioning as they ought to. Entities within the world reveal their Being while they are functioning as they are predestined to be. Dasein is predestined to comprehend, interpret and care for the Being of the entities in the involvement with other beings. For instance, a table is functioning as table while people are eating their meal at it. As Dasein, the child is experiencing the functioning of the table being used by the adults. The table being used by the adults reveals what it is (the Being of the table) to the child, and the child simultaneously understands the Being of the table. In language learning, the name label is not so significant as the understanding of the Being of the table, the merge of which with the vocal symbol becomes our language with the understanding aspect becoming the meaning of language and the symbol the form (in Saussure's terminology, the "signified" and the "signifier" [11]). This is why Heidegger views human language as the "house of Being."

The comprehension of the Being of things serves as interpretation and belief for them. When a child is asked to come to the table to have his meal, he may refuse to move and say that the stool is his table. This shows that he has grasped the Being of the table. The Being of the table is its function to support the performance of having meals. This experience of the Being of the table not only enables the child to have the knowledge of the Being of the table, the world of the table, including the relationships between the containers of the meal and the surface of the table, and between the people and the table, but also makes him believe that the thing that functions as the table that he has experienced is the table.

The experience of the Dasein also serves as the underlying value assessment parameters for future interaction with the outside world. For instance, if you had the experience of being bitten by a dog, then you would know that dogs can bite people even though you were bitten by a particular dog. Moreover, you would develop the feeling of being afraid of dogs. This kind of memory is technically called 'episodic memory' or 'biographical memory' in psychology. According to Hoerl [12], episodic recollection, in some sense, has become a common theme in 
recent literature [13-14]. Episodic recollection involves "re-experiencing" or "re-living" what one has experienced as the events had happened. Mahr \& Csibra [15] maintain that humans are obsessed with reminiscing about past experiences and sharing those experiences with others, and that psychologists have identified the basis of this obsession as originating in episodic memory.

Now we shall return to the scenario Engels discussed in his thesis, where the men in the making had something to say to their peers in the process of labor. Let us imagine that some of them (or one of them) had seen a group of lions chasing a group of deer. (This might be a very common scene for our ancestors.) The communotion is the experience stored in the mind as an episodic memory.

Undoubtedly, the humans at the outset of language evolution did not have names for the lions and deer they had seen. But once they experienced the lions chasing the deer, they knew what they were, though their knowledge of them might be different from ours at the present time. Their knowledge bears comparison with that of an infant who has seen lions chasing deer for the first time in a movie about the animal world. The knowledge of the modern civilization has increased in the same fashion, and our language has evolved simultaneously alongside the evolution of human civilization. Human language evolution is in reality the history of naming the knowledge derived from humans' direct experience of the outside world. As the experiencing of the outside world is also a process of understanding based on the sensorimotor capacity, cognitive faculty and the pragmatic value system, experiences that have undergone such an understanding process turn into human knowledge. Therefore, when the men in the making had evolved to the point where they had something to say to each other, the something in their mind was not just simply an episodic recollection, it was something intended to share with the audience for a certain communicative purpose. This function of the episodic memory serving the purpose of human communication is what Soteriou claimed to be the "epistemological role of episodic recollection [16]."

In human experience, things unveil their Being to humans, who comprehend and interpret them with their innate capacities and value beliefs. In the case of the lions chasing the deer, humans came to know what lions, deer and chase meant by experiencing the event, even though they did not have names for them. An event is a happening, or an occurrence in a certain time and space, perceived and comprehended by a particular individual with innate biological and cognitive capacities, and with prior experience serving as insights for discernment. Comprehension of the experience involves understanding the relationships between the elements in the event. Thus at the point where our ancestors had something to say to each other, they could understand that the lions were the stronger party and deer were the weaker, and the stronger would kill the weaker. If the lions were coming to them, they would run or hide or fight. They would also know, by taking measure of the deer, that the deer might be their hunting target rather than the lions.

With the elaboration on the something in the mind of our ancestors at the point of social communication, we conclude that the something came from our ancestors' experienced event that was recollected as an episodic memory that they intended and were impelled, with a burning desire, to say to each other, and that the experienced event had already unveiled to them the nature of the event and the relationships between the components involved in the event. With the instance of the lions chasing the deer, it is safe to say that the communotion was the visual perception and mental conception of the episodic memory retained in the mind and suspended in the consciousness beckoning the symbolic means to get expressed. The primary structure of the event is: the lions $\rightarrow$ chase $\rightarrow$ the deer. No matter how a particular 
language conventionally verbalizes the event, the primordial episodic picture remains the same.

This analysis and interpretation of the communotion also holds true for our daily conversation. When examining the relationship between thought and speech, Vygotsky [7: 251] made an insightful description of the heterogeneous relation between the communotion and linguistic expression:

Thought, unlike speech, does not consist of separate units. When I wish to communicate the thought that today I saw a barefoot boy in a blue shirt running down the street, I do not see every item separately: the boy, the shirt, its blue color, his running, the absence of shoes. I conceive of all this in one thought, but put it into separate words. A speaker often takes several minutes to disclose one thought. In his mind the whole thought is present at once, but in speech it has to be developed successively. A thought may be compared to a cloud shedding a shower of words. Precisely because thought does not have its automatic counterpart in words, the transition from thought to word leads through meaning. In our speech, there is always the hidden thought, the subtext. Because a direct transition from thought to word is impossible, there have always been laments about the inexpressibility of thought.

The thought that Vygotsky discussed in the above statement is the communotion in this paper. Regardless of the language and culture background, all people with eyes, even animals such as dogs, could see the running boy. In a certain communicative context, the episodic memory of this experience might become the communotion for expression. The Dasein with the event altogether became the experience of the viewer, and this experience includes the time, the place, the boy, the running, the feet, the face, the shirt, the trousers, the crowd, the building, etc. All human particular languages have evolved for the expression of this human experience, and the natural logic and structures with regard to the elements and their attributes of the event underpin the conventionally verbalized particular languages of humans.

The same is also true of infants in the pre-language stage. Bloomfield [8: 29-31], the famous American linguist, made very careful observations about an infant learning English. Within his academic milieu, he was unable to conduct any cognitive analysis. Nevertheless, his behaviorist discussion itself reveals the actual fact of native language acquisition of infants. The following is his description about the process of an infant learning to speak English.

(1) Under various stimuli the child utters and repeats vocal sounds. This seems to be an inherited trait. Suppose he makes a noise which we may represent as $d a, \ldots$

(2) Some person, say the mother, utters in the child's presence a sound which resembles one of the child's babbling syllables. For instance, she says doll. ... Grown-ups seem to have observed this everywhere, ....

(3) ...the child forms a new habit: the sight and feel of the doll suffice to make him say $d a$... it may not sound like any of their words, but this is due merely to its imperfection. It is not likely that children ever invent a word.

(4) The habit of saying $d a$ at sight of the doll gives rise to further habits. ... if one day the mother forgets to give him the doll, he may nevertheless cry $d a$, $d a$ after his bath. "He is asking for his doll," says the mother, and she is right, since doubtless an adult's "asking for" or "wanting" things is only a more complicated type of the same situation. The child has now embarked upon abstract or displaced speech: he names a thing even when that thing is not present.

(5) The child's speech is perfected by its results. ... if he says his $d a$, $d a$ imperfectly ... then his elders are not stimulated to give him the doll. ... At a much later stage, if he says 
Daddy bringed it, he merely gets a disappointing answer such as No! You must say "Daddy brought it"; but if he says Daddy brought it, he is likely to hear the form over again: Yes, Daddy brought it, and to get favorable practical response.

Bloomfield's observation can be reinterpreted, using the theory of the communotion. (1) The child has innate propensity and capacity to imitate human sound, and it would be a long period of time for him to perfect his pronunciation. (2) Before the infant learns to say $d a$, he has experienced (seen and felt) the doll and kept it in his mind as an episodic memory. Next time he sees it, he must feel familiar with it. That is to say, before he learns the name of the doll he already has it in his mind. The sound $d a$ is to label the doll that he has experienced. (3) Language learning needs repetition. The infant cannot remember the association of the sound $d a$ with the doll by experiencing it only once. It is necessary that the mother repeats it many times for the child to learn the name. (4) The most significant part of the observation is the crying of the infant after the bath one day, when his mother forgets to give him the doll. In the theory of the behaviorist psychology, it is the habit of the infant that impels the boy to cry. Yes, it is a habit, but it is mental and psychological habit. The bath is associated mentally with the doll. When the bath ends, the doll should ensue, which is what he expects and desires to see. At this very moment, the communotion pops up. This is just like a person who has formed the habit of smoking after a meal. If one day after a meal he finds that there are no cigarettes on the table, he would like to ask his wife for them. The infant's cry is caused by the desire of the communotion, which will later become the target of language expression in the process of language acquisition. The communotion will gradually materialize and become verbalized into something like I want the doll or would you please give me the doll. (5) Language acquisition involves not only the representation of the concepts in the communotion by means of symbolic vocal sounds, but also the convention of the rules in the construction of the relationships of the components of the communotion through symbols of vocal sounds. The conventional phenotype of the relationships between the components of a sentence is in reality the manifestation of the genotype of the relationships of the components of the communotion.

To recapitulate, human language has evolved for, from and with the communotin, which itself evolves from prior experience and pops up with the intention to communicate with others something relevant to the experience. Language does not evolve for the experience, or the cognition involved in the experience. Experience and cognition do not necessarily entail language. Only when the experience becomes the episodic memory and emerges as the communotion, the target of expression, under the pressure of social communotion, can the evolution of human language take place.

The scenarios described above are ubiquitous: the experience is ubiquitous; the communnotion is ubiquitous; the Dasein's world is ubiquitous; the relationships in the "lions chasing deer" in the experience are ubiquitous; the manners of human perception and conceptions of the relationships are ubiquitous; the mechanism of human communication is ubiquitous; and the mechanism for the merging of the meaning from the communotion and the vocal sounds from the speech organs is ubiquitous. What is not ubiquitous is the conventional verbalization and materialization of the communotion. But no matter how a human particular language verbalizes the elements and their structures of the communotion, the primordial and prototypical structures of the communotion remain the same. This is the source of the universality of all human particular languages.

The following part is intended to illuminate the conventionalization and verbalization of the communotion by analyzing the phenotype of the Chinese language in comparison with the English language. It is hoped that the illuminating discussion will make it clear that the 
hierarchical structures of the elements and relationships between the elements and their attributes in the communotion are the universal and common source for all human particular languages and that the English language merely represents one typical mode of conventionalization and the Chinese language another typical mode.

\section{DESIGN PRINCIPLES UNDERLYING THE EVOLUTION OF THE SYNTACTIC PROPERTIES OF THE CHINESE LANGUAGE}

All human particular languages have evolved for, from and with the communotion of the particular cultures created by particular communities. This is not to say that people in different cultures have experienced totally different outside natural and sociocultural worlds, or that they have totally different innate capacities and faculties to perceive and comprehend the world they have lived, but rather that they have construed the experienced and lived world in different ways, and that when they created their symbolic tools of language, they encoded in the symbols their construal and understanding and feeling of the nature of the things and events they had experienced. Halliday and Matthiessen [17] discussed the construal of experience through meaning. It is true that in a mature culture with its language, the experience of the world is construed from meaning, but it is wrong to apply this view to the study of human language evolution, simply because, for our ancestors without any language and prefabricated meaning at hand, the opposite is true that meaning is first construed through experience and then merged with vocal sound into language. In the course of verbalization of the communotion, different cultures have developed their unique traditions of verbalization, which function as an internal force driving different languages forward to the present phenotypic properties of the particular languages.

To put it briefly, all human particular languages have evolved upon the mechanism of verbalization and expression of the communotion, which derives from the lived experience through episodic memory and presents itself by the intentionality and desire for communication. Based upon these mechanisms, different particular languages have evolved, complying with the design principles that have been geared towards the phenotypes of the particular languages.

The study of the evolution of human language from the viewpoint of the communotion verbalized by the tool of the symbolic vocal sounds for its expression enables us to examine human language from the following perspectives: the perceptual and conceptual perspective that views the evolution of human language as naming or signifying process that encrypts the elements that are cognitively isolated or carved out from the wholeness of the experience; the communotional and functional perspective that sees the signified and verbalized symbolic vocal sounds as a tool that equips humans with an external means to express the internal communotion during the process of social communication; and the conventional perspective that regards human language as cultural linguistic construction from the basic conventional rules for the design and arrangement of the vocal sounds, to the rules for phrasing, and for the syntactic properties.

This three-level hierarchical evolutionary process of human language can be illustrated with Vygotsy's example mentioned in the above section: [T]oday I saw a barefoot boy in a blue shirt running down the street. This sentence originates from the experience of the witness. Every normal sighted person, regardless of his language background, could remember the event if he happened to see it. (Doubtless some nonhuman animals could also see it and remember it.) Vygotsky's example can be rendered as Fig. 1 (the conventionalized English versions from the basic elements of the communotion): 


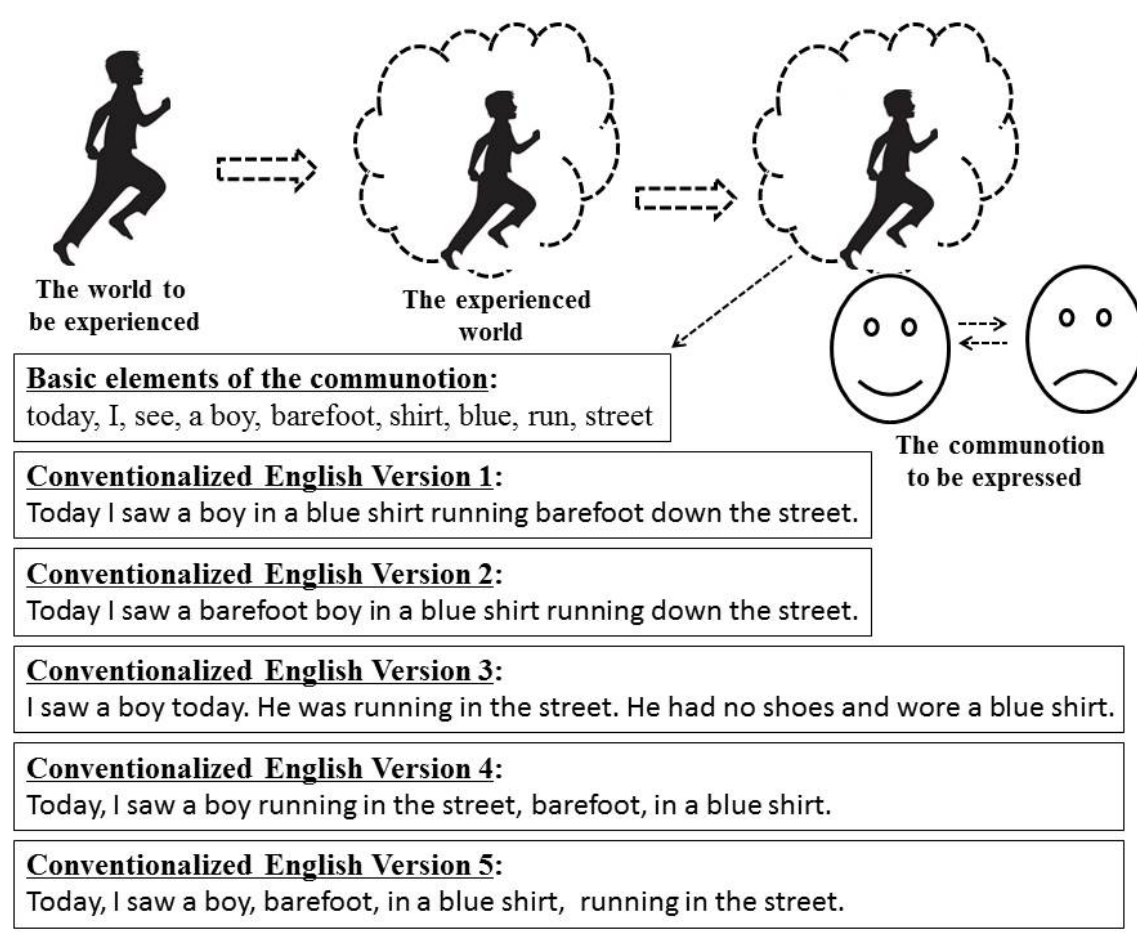

Fig. 1 The conventionalized English versions of Vygotsky's Example

Fig. 2 is the conventionalized Chinese versions from the basic elements of the communotion (the English under each Chinese sentence is word-for-word literal translation of the Chinese):

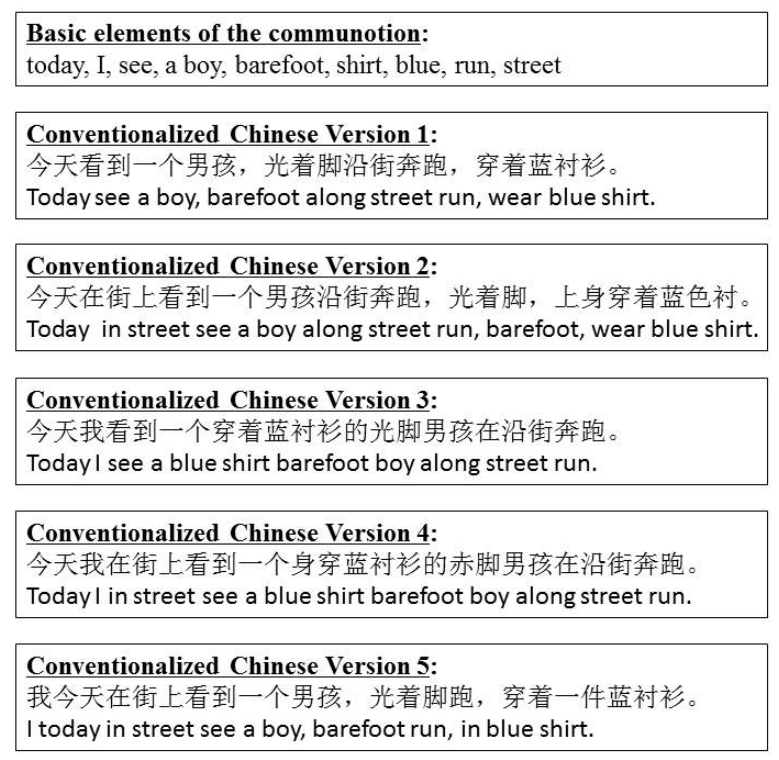

Fig. 2 The conventionalized Chinese versions of Vygotsky's Example

All the versions, English and Chinese, are grammatically correct sentences. The relationships between the elements (parts) of the sentences are in reality those between the components of the communotion. The event is encapsulated in what I saw as Dasein. What I saw happens at the time of today and the place of the street. The running is done by the boy; barefoot (an attribute) is the description of the boy's feet and is focalized because of the relevance to running; and the blue shirt (an attribute) belongs to the boy and is part of the description of the boy. From the theory of Dasein, all elements in the experience are naturally and logically situated in the event. At the very moment of communication, the whole episodic memory appears in the consciousness and intentionality as an intended target of expression. 
As for the acceptability and typicality of the different versions of both English and Chinese, the English Versions 1 and 2, and the Chinese Versions 1, 2 and 5 might sound more normal and typical. The English Versions 3 to 5 might seem fragmental, and the Chinese Versions 3 and 4 might seem syntactically Westernized. The comparison between the English and Chinese versions enables us to become aware of the differences between the two languages. The most conspicuous differences in the phenotypes of the two languages are the arrangement of the components of the communotion and the conception of the sentencehood.

The English language conceives a sentence as a complete syntactic structure, typically containing a subject, a predicate and, sometimes, some other additional elements, or in a compound and complex sentence, containing a main clause and sometimes one or more subordinate clauses. The verb of the sentence is finite, indicating such grammatical categories as tense, person, and number. In the written form, an English sentence typically begins with a capital letter of the first word and ends with a punctuation that indicates the end of the sentence. In speech, it shows a recognizable intonation pattern, and with relatively long pauses between sentences. Briefly, the English language is syntax-based, structure-bound and rulebound.

In contrast, the Chinese language is image-based, relatively structure-free and rule-free. First of all, the so-called subject of the sentence (in the above sentence, the subject is $I$ ) can be omitted. As an interlocutor in a dialogue, one does not need to say I see or I saw. It is the latent rule in Chinese: if it is clear that the subject is the speaker in the context or that the action is done by the speaker, then it can be omitted. Hence today see a boy run down the street. (Of course, if the subject is emphasized, it cannot be left out. It is $I$, not anybody else, that saw the boy running.) In English, the subject of you is conventionally omitted in imperative sentences, but in Chinese all the subjects can be conventionally left out when the context is obvious and unambiguous. Second, there is no need to have the grammatical category of tense as a grammatical rule, since the time today and the event itself have indicated the time when the action of see took place. Then why bother changing the form of the word see to saw (an irregular verb)? Third, the rigorous rules at the phenotypic level can only impede the process of expressing the communotion. In the context, all the descriptions, barefoot, blue and run, refer to the boy. Then why bother adding in, the and -ing. These phenotypic features are merely man-made cultural conventions by the English community (or derived conventions for other languages) in the development of the English language, not at all the manifestation of the genotypic nature of the communotion. Fourth, the Chinese language focuses on the image of the communotion, depicting the episodic scene of the event in the memory. When we depict or describe an event, we usually set up the backdrop of an event by first introducing the time, space and main character. Hence, in Chinese, the typical order of the depiction of an event is: time + place + main event + description 1 of the event + description 2 of the event + description 3 of the event ... (following a certain logical order, e.g. from the upper part to the lower, from the left to the right, from the near to the far or from the far to the near). Hence, in Chinese, the typical structure of Vygotsky's example is: jintian (today) [TIME] zai jie shang (in street) [PLACE] kan dao (see) yige nanhai (a boy) yanjie benpao (running down street) [MAIN EVENT], guangzhe jiao (barefoot) [DESCRIPTION 1], chuanzhe lan chenshan (wear blue shirt) [DESCRIPTION 2].

In the above discussion, I gave an ontological and genotypic account of the phenotypic syntactic structures of the Chinese language from the perspective of the theory of communotion. The phenotypic structures of Chinese are confined not merely to syntactic structures, but also to the depicting or descriptive structures of the communotion. Hence, the 
Chinese language is not amenable to the frame of reference of the syntactic analysis in the Western tradition. (The next section will elaborate on this point.)

Ontologically speaking, the communotion to be expressed is merely a mental, psychological, stereoscopic, episodic image. This type of episodic image has been universally construed by humans using their perceptual, cognitive and logical faculty based on their pragmatic value system. There are actually two possible ways to transduce it into the verbalization of linear symbolic language of vocal sounds: structure-based verbalization and image-based verbalization. Structure-based verbalization is designed to encapsulate the relational elements of the communotion in a rule-governed hierarchically structured sentence, whereas imagebased verbalization is devised to delineate the mental episodic image through a sequential process in a whole-part and point-to-point fashion. In the example given by Vygotsky, the image of the boy was running along the street is the main part of the event, and the description of the feet, the blue shirt or some other parts is secondary.

From these properties of the Chinese language, made salient in comparison with the English language, we can formulate the design principles underlying the evolution of the Chinese language.

\section{Principle of Situatedness}

Human language has evolved for, from and with the communotion, which in turn originates from the speaker's experience and automatically pops up upon the speaker's recognition of the context in social communication. The Principle of Situatedness is inherently and intrinsically embedded in the working mechanism of the verbalization transformation from the communotion to the phenotype of the Chinese language. Speech, the production, generation and use of human language, is situated in the process of communication. It is a dynamic process of self-adjustment to the image of the communotion on the one hand and, on the other hand, to the here-and-now context of the on-going communication. Human communication is ontologically and functionally intertwined with the internal, mental, psychological, personal experience and the external, physical, sociocultural, communicative world. As such, the symbolic tool of language should be best understood as serving the purpose of communication through the meaning image of the symbolic language construed from both the perspective of the internal personal experience and the perspective of the external context of the situation of the interlocutors.

In the process of social communication, our internal mental mechanisms are manipulated by our autonoetic consciousness to make self-judgment based on the resource gained from the internal and external worlds of the interlocutor. The traditional cognitive science characterizes the mind as the internal entity that has the faculty of computation and recursion, whereas the perspective of situated cognitive theory emphasizes the working mechanism of the mind interacting with the inner personal experience and the outer communicative situation.

The Principle of Situatedness states that human language originates and has been situated in human sociocultural communication, and that, functioning as a symbolic tool for the verbalization and expression of the communotion, it makes full use of the internal context of the experience, the on-going context of the conversation, and the external context of the situation and atmosphere concerning the interlocutors. The Principle of Situatedness applies to all human particular languages, but the Chinese language perfectly exemplifies this principle with its basic properties of being image-based, structure-free and rule-free. 
The following Chinese conversation between husband and wife can best illustrate this Principle of Situatedness. The husband got home and found his son had not come back from school. (This is a typical interface between the internal context of the husband's personal experience and the external situation where his son should have been present, resulting in the generation of the communotion.) He asked his wife (the communotion and intention of asking his wife about his son began to take shape the moment he saw his wife and realized that his son was not home):

Husband: Erzi ne? (Son? The character ne in Chinese is a modal word used at the end of a sentence to ask a question. The husband presumed that his wife should know it was their son that he was referring to. So the word son has no modifier.)

Wife: Fangxue huilai wo rang ta qu kan yeye le. (Returned from school. I asked him to see Grandpa. The wife, with her internal context of personal experience, and transcontext knowledge, knew who the son was, and knew what her husband intended to know. Therefore, she told her husband that their son had come back from school and she wanted him to see his grandfather.)

Husband: Zenme le? (What happened? Both the husband and the wife knew that Grandpa was old, and had not been well. So the husband wanted to know what happened to Grandpa. In the context, they both knew that it was Grandpa, not the son, that the husband was referring to when he asked what had happened.)

Wife: Wanshang zong kesou. (Coughed all night. In this context, it is unnecessary to point out who coughed all night.)

Husband: Kan yisheng le ma? (Seen doctor? Again, the context has made it clear that the husband was referring to Grandpa.)

Wife: Kanle, geile dian yao. (Seen. Gave some medicine. Here the wife echoed the word seen in her husband's question, so it refers to Grandpa. But in the context, only the doctor could give medicine. Hence the doctor can also be left out in the context.)

Husband: Chile ruhe? (How after taken? Both the husband and the wife knew that if the doctor gave the patient some medicine, the patient was supposed to take it. So the husband did not need to mention who took the medicine. What the husband cared about was the effect of the medicine after Grandpa had taken it.)

From this mini-talk between husband and wife, we can see clearly how the talk in Chinese is situated in the context of their mutual and individual knowledge gained from their personal experience and in the on-going conversational context which consists of the physical interaction between the husband and wife and the part of the talk they had already undertaken.

When we say that the Chinese language is an efficient language, we mean that it makes good use of the implicit and explicit contexts. It is the doctor who gives medicine and it is the patient who sees the doctor and takes the medicine. Hence, in the context of on-going conversation, it is simply unnecessary to mention grandpa or the doctor. The Chinese language is also flexible since these so-called subjects can be added when necessary, but it would be redundant if the context is evident.

In an article discussing Chinese logic and the Chinese language, Chao [18], a well-known linguist of the Chinese language, illustrated the spirit of Chinese logic in the subject-predicate relation with the freeway sign in South San Francisco: Third Street Keep Right. He said that the grammar of ordinary English would naturally treat such a form as an ellipsis of: For going to Third Street, drivers should keep to the right. Or treated as an imperative: Going to Third Street, keep to the right. He concluded that "a much larger percentage of Chinese sentences, not 
on road signs, but in deliberately articulated discourse, are of the type where the subject is not the agent of the word for the action in the predicate."

\section{Principle of Theme Depiction}

If language production is viewed as the verbalization and expression of the communotion, then the production is the narration of the episodic story of the communotion. The story is the communotion that an artist, or a writer, or a poet, or a critic, or a commentator attempts to convey to others through the tool of language or some other symbols. Putting the story of the communotion into linear language of narration requires conventional and logical structures. They are conventional because they are collectively designed, recognized and observed; they are logical because these structures or procedures are, in a sense, universal to mankind. The story of an event is situated in a background, unveiled through time and space and following the cause-effect plot. As was discussed earlier in the section of the communotion theory, the communotion itself is composed of different elements and these elements have different attributes. The presentation of the whole of the communotion and its elements necessitates rational and reasonable arrangement. The mechanisms of human cognition are also universal, functioning on faculties that were shaped through the evolutionary process of human interaction with the natural and sociocultural world.

In order to tell the story of the communotion through the linear language, the speaker or writer will have to arrange the story of the communotion in such a sequential linear order that the hierarchical relationships between the elements and between the whole theme of the story and minor themes can be displayed through the depiction of the story. Since the story of the communotion is situated in the storyteller's experience or imagination as an episodic event, it is quite natural to start the story by introducing the background of the story or the topic of an argument. This is the normal arrangement in story or fiction writing, and, as it were, for any kind of writing.

Such is quintessentially the Chinese language at the level of minute theme depiction. In Vygotsky's example: jintian (today) kandao (see) yige (a) nanhai (boy), yanjie (down street) benpao (run), chijiao (barefoot), shen chuan (wear) lan se (blue) chenshan (shirt). (Today [I] saw a boy in a blue shirt running barefoot down the street.)

The word today functions as the time background of the story of the communotion that I intend and attempt to tell you. The phrase see a boy serves as the topic, and run down the street, barefoot and wear a blue shirt are the minor themes, i.e. further depictions, of the boy.

The Principle of Theme Depiction can be better illustrated by ancient Chinese. Take as an example The Classic of Regions Beyond the Seas: The North, a Chinese classic work written about 2000 years ago. Versions of the text existed in the $4^{\text {th }}$ century BC. It is a compilation of mythic geography and myths.

钟山之神，名曰烛阴，视为昼，眠为夜，吹为冬，呼为夏，不饮，不食，不息， 息为风；身长千里，在无䁈之东，其为物，人面，蛇身，赤色，居钟山下。

The following is word-to-word literal translation into English regardless of the English conventional grammar:

Deity of Bell Mount, named Torch Shade, eyes open, daytime; eyes closed, night; blow, winter; exhale, summer; not drink, not eat, not breathe; if breathe, then wind; body length a thousand miles; in east of Wuqi; he is non-human, human face, snake body, scarlet, live at foot of Bell Mount. 
English translation:

The deity of Mount Bell is named Torch Shade. When the deity opens his eyes, there is daylight, and when he shuts his eyes, there is night. When he blows, it is winter, and when he exhales, it is summer. He neither drinks, nor eats, nor breathes. If he does breathe, there is wind. His body is a thousand miles long. He is in the east of the country of Wuqi. He is non-human. He has a human face and a snake's body, and he is scarlet in color. He lives at the foot of Mount Bell.

In this narrative, the deity of Mount Bell is the focalization of the theme or the topic of the communotion intended to be depicted. Logically speaking, the deity of Mount Bell is the subject, and all the rest is the predicate (the terms of predicate and subject are used here as logic terms, not grammatical terms), giving detailed information about the subject. According to the Principle of Situatedness, since the deity of Mount Bell serves as the main subject and hence the context, it would be unnecessary to indicate the subject of each of the predicates in the successive depiction. According to the Principle of Theme Depiction, the deity of the theme includes many predicates, various attributes that can be depicted. These predicates should be conventionally and logically arranged. For instance, the name should be first mentioned; eyes open before eyes closed; daytime before night; winter before summer; and drink before eat.

\section{Principle of Dynamic Focalization}

This principle is related to the Principle of Theme Depiction. While the Principle of Theme Depiction states that the Chinese language has evolved following the pattern of theme depiction of the communotion, the Principle of Dynamic Focalization indicates the manner of the theme depiction. The depiction of themes requires dynamic shifts of focus of the depicting process, from the major theme(s) to the minor theme(s), or from one minor theme to another. In the above example, the theme depiction moves from the deity of Mount Bell as a whole to its varied predicates, and from the predicate of name to the predicate of day and night, to the predicate of winter and summer, etc. In other words, the speaker, as a narrator, concentrates his mind's eye on different thematic points of the elements of the communotion intended to convey to the audience.

If the focus of the narration of the communotion is upon the themes, then evolution of the language must have centered around the arrangement of the themes, leading to the convention and tradition of phrase-based structures (rather than sentence-based) and sequences of notions. Therefore, in the Chinese language, the concept of a sentence refers to the completion or fulfillment of a theme or a group of related minor themes, not the completion of a syntactic structure. The following is a typical example. It is from the novel Red Sorghum (Section 5) by Mo Yan, the Nobel Prize winner in literature in 2012.

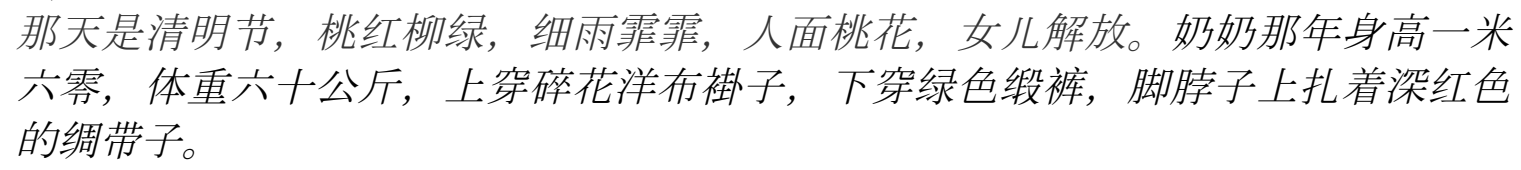

It was Qingming, the day set aside to attend ancestral graves; peach trees were in full red bloom, willows were green, a fine rain was falling, and the girls' faces looked like peach blossoms. It was a day of freedom for them. That year Grandma was five feet four inches tall and weighed about 130 pounds. She was wearing a cotton print jacket over green satin trousers, with scarlet bands of silk tied around her ankles [19].

This description consists of two sentences in Chinese ending with the punctuation mark " 。”, and the mark "," within the sentences functions as pauses for the dynamic shifting of focuses 
from one minor theme to another under one main theme. The theme arrangement can be best illustrated by Fig. 3 and Fig. 4:

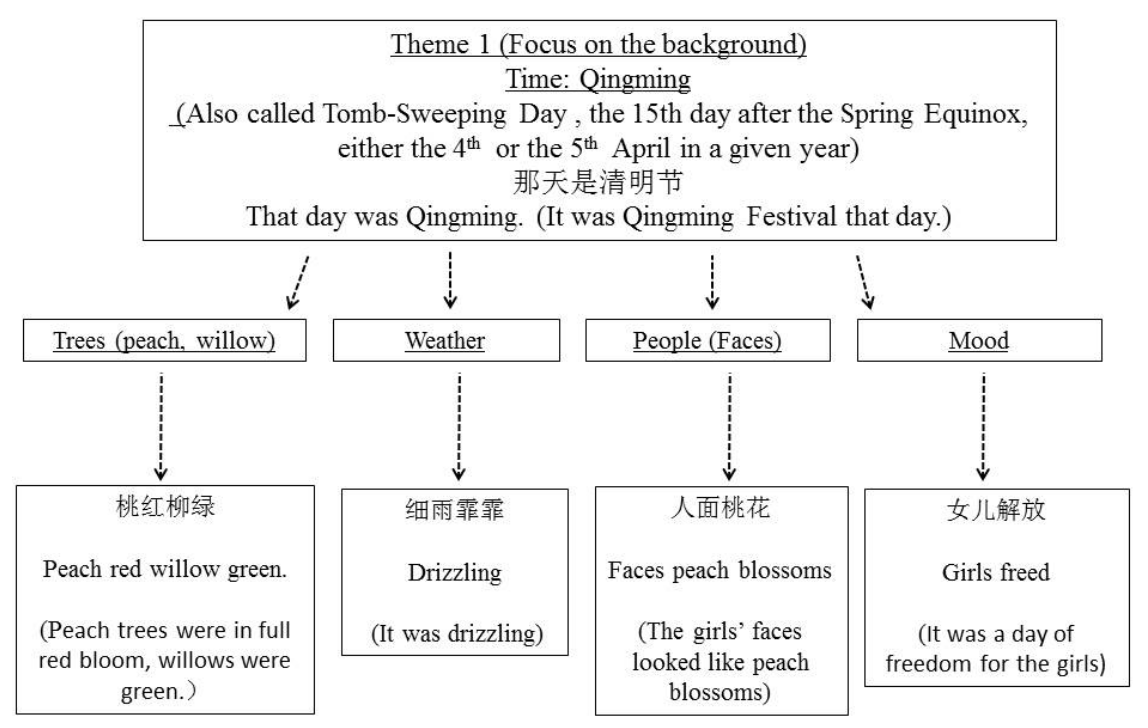

Fig. 3 Mo's Depiction of the Background Qingming

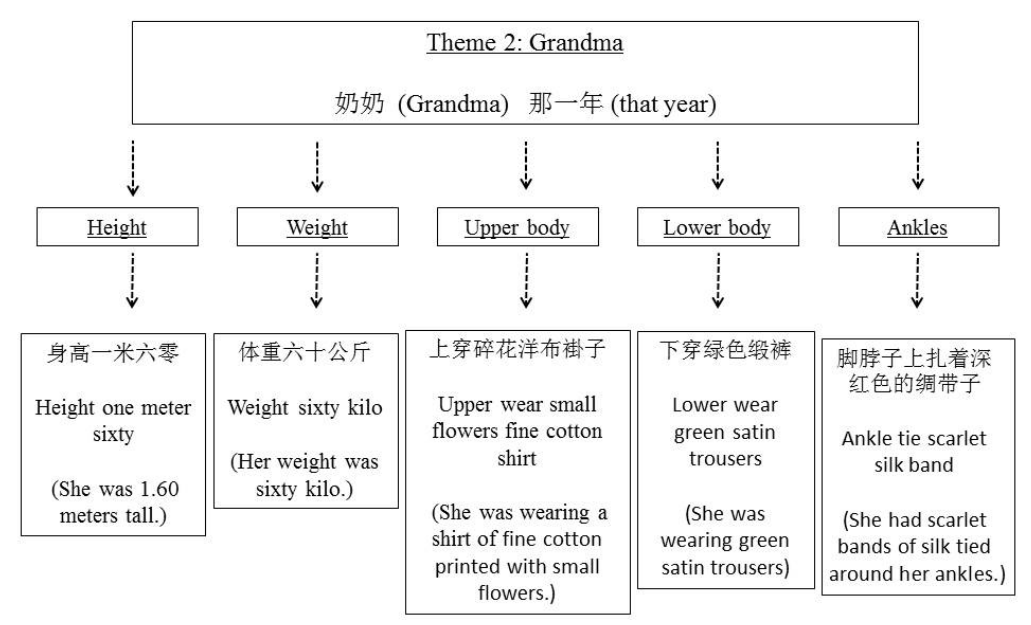

Fig. 4 Mo's Depiction of Grandma

In this part of the depiction, there are two main focal themes (Theme 1, the background and Theme 2, the character of Grandma), which are encapsulated in two Chinese sentences (the Chinese concept of sentencehood). It is clear that the purpose of the depiction of Theme 1 the background is to highlight Theme 2 the character of Grandma. This logic for the sequential narration, the depiction of the background before that of the character, is natural and universal in the narration of events or stories in all cultures.

Qingming Festival in Chinese culture is the day for people to attend their ancestral graves. But it is also a time to appreciate the beautiful scenery of spring after a long winter in North China, when peach trees blossom and willow trees sprout. The weather at this time of the season is characterized by drizzles. The trees, the weather, the happy and beautiful smiles on the faces of 
the people and the feeling of freedom of the girls on rare occasions to come to the outside in the nature, all these find their niches in Qingming. Peach trees and willow trees are parallel to each other, but in the conventional development of the Chinese language, peach trees are before willow trees in position and the color red before green. The four minor themes under the main theme of the background of Qingming are parallel in function but sequential in importance. Each minor theme has its own focus of topic or subject and its predicate(s). In the conventional development of the Chinese language, this typical mode of depiction developed into the convention of parallelism that contains the same number of Chinese characters with the typical one being four Chinese characters (four syllables in pronunciation, each syllable being a word), like the one in this depiction. The beauty of the Chinese language lies in this kind of parallelism and the tonal sound of the same number of syllables. Please appreciate: Táo hóng liǔ lǜ (peach trees were in full red blossom, and willow trees were green), xì yǔ méng méng (it was drizzling), rén miàn táo huā (the girl's faces looked like peach blossoms), nŭ́ ér jiě fàng (it was day of freedom for the girls).

Likewise, Theme 2 focuses on the topic of Grandma that year, the depiction of whom includes five parallel minor themes. Again, the sequence of these parallels are not randomly arranged, but logically and conventionally ordered. The depiction of height is before that of weight, and the description of the appearance of a person usually starts from the upper part of the body to the lower part of the body and then to the waist or other parts of the body. Hence we have the sequence of height, weight, the upper part of the body, the lower part of the body, and the ankles.

Human language did not evolve for itself, but for the expression of human communotin. Since human communotion contains Dasein's experience, the depiction or narration of the experience is basically natural, logical, and universal to mankind. However, since a particular language of a certain culture developed with its social, ideological and literary concepts, and the primordial development would generate a certain internal conventional force that might drive the development forward along certain direction and the result would become the basic properties of the language.

\section{Principle of Phrase-Based Construction}

One of the corollaries of the Theme Depiction and Dynamic Focalization is the development of the phrase-based property of the Chinese language. As early as the 1980s, Dexi Zhu [20] elaborated on the phrase-based phenomenon of the Chinese language. This article attempts to identify the ontology of the phrase-based property. As was mentioned above, the minor themes are the predicates of the subject (the main theme), and the predicates relate to the parts and attributes of the subject, and these parts and attributes themselves might be composed of subsubjects and sub-predicates. Therefore, the depiction of these minor themes entails the description and arrangement of the relationships of the sub-subjects and sub-predicates in the parts and attributes of the main theme.

Since all the minor themes function as the predicates of the subject, they are parallel in function and could be rhetorically beautified into parallel structures. The theme depiction and the dynamic focalization of the minor themes of the two main themes, the background and Grandma, serve as perfect examples for the illustration of the Principle of Phrase-Based Construction. If you see two security guards at the gate yesterday, wearing riot helmets, leather boots, and uniforms with armed belts, then according to the principles of Situatedness, Theme Depiction, Dynamic Focalization and Phrase-Based Construction, this communotion can be rendered as: Today at the gate (theme of background of time and place) see (subject is omitted 
because of situatedness) two security guards (main theme), head (the upper part of the body, the first depiction in the sequence of the depiction of the minor themes) wear riot helmets (sub-predicate of the sub-subject of the head), feet wear leather boots (the lower part of the body, in the Chinese convention of depiction, the upper is the opposite of the lower, and hence the sequence: the upper, the lower and the middle), body wear uniforms, waist wear armed belt. Fig. 5 below shows the mental depiction of the episodic memory in the communotion:

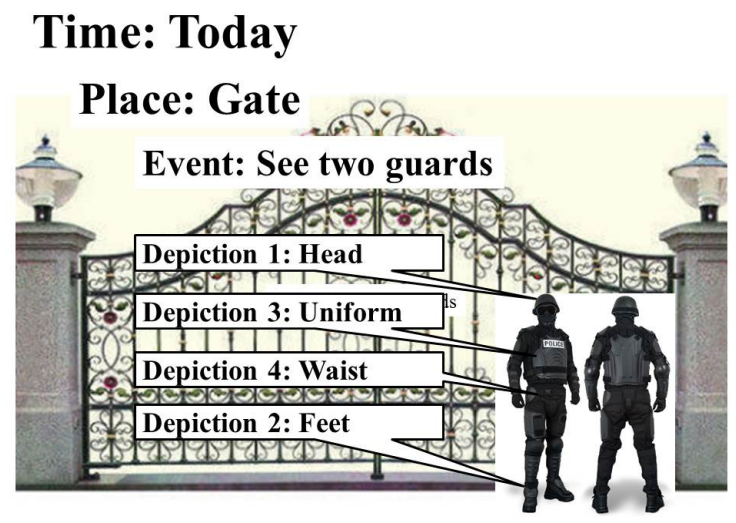

Fig. 5 The Example of the Two Security Guards

As Chinese is a monosyllabic and tonal language, the four body parts are four monosyllabic nouns: tou (head), jiao (feet), shen (body), yao (waist); and the four actions relating to the articles worn by the sub-subjects (Chinese have different concepts for the actions of wearing hats, shoes, clothing, and belt): dai (head-wear), chuan (feet-wear), zhuo (body-wear), and ji (waist-wear, tie) are also four monosyllabic verbs. Hence, a beautiful parallel structure has been formed. The normal and typical structure is: monosyllabic noun + monosyllabic verb + monosyllabic adjective + monosyllabic noun.

\section{Principle of Word Order}

The Chinese language patterns itself on the episodic image in the communotion. The image of lions chasing the deer presents itself on the structure of lions + chase + deer, just like a real picture of lions chasing deer. This principle can also be explained by the Principle of Dynamic Focalization: the mind's eye moves with the running of the animals. The sequential order of the linear language contains the hierarchical structure of the natural and logical (or semantic, after the communotion is verbalized) relationships of the components of the communotion. The Principle of Word Order demonstrates not only the sequential order of the elements of the communotion (e.g. wo da ta, I beat him; or ta da wo, he beat me), but also the order in the construction of the phrase-based structures according to the Principles of Theme Depiction and Theme Focalization. The examples given above serve as perfect examples to illustrate the Principle of Word Order. For instance, theme background (time + place, time before place): yesterday + at the gate; the event (the focal theme): see two security guards (guards are the focus); minor themes (the depiction of the two guards: body parts + depiction): head + feet + body + waist. A Chinese language speaker would follow this sequential order of dynamic focalization: the time (yesterday) + the place (at the gate) + the event (see two security guards): the head + predicate; the feet + predicate; the body + predicate; the waist + predicate. In general, three key minor principles can be identified regarding the sequential order of the components of the communotion in the dynamic focalization of the theme depiction. All these properties might have naturally evolved from the Chinese people's perception and conception of the relationships of the components of the communotion intended to convey to the audience, and conventionalized in the Chinese language. 
The first minor principle concerning the sequential order is the priority of the main theme over the minor theme(s). This property can be easily seen from the examples discussed above: the boy before his attributes; the deity of Mount Bell preceding his name and other predicates; Grandma followed by depictions of her attributes of height, weight, dresses of the upper and lower body and the ornaments on the ankles; and the security guards prior to the their attributes. This principle can also extend to the principle of order pertinent to the depiction of time, space, etc. For instance, the typical order of time and space depiction in Chinese is from the big to the small. In English, it is normal to say "there is a meeting about the name change of the school in the meeting room on the second floor of the main building at 5:00 in the afternoon next Tuesday." In Chinese, the English sentence can be rendered like this (following the English convention of putting everything in one sentence): next Tuesday + afternoon + 5:00 + on the second floor + in the meeting room + there is $+a+$ about the name change of the school + meeting.

The second principle is the priority of the background of time, place, circumstance, or condition over the thematic event itself. Hence the order: next Tuesday + on the second floor of the main building + there is a meeting; today + see a boy; yesterday + at the gate + see two security guards; and eyes open + day time, eyes close + night.

The third principle is the sequence of modifier before the modified. The modifier can be anything that is pertinent to the central word to be modified, and the modified can also be anything, but typically people, things and actions. Sometimes 的 (de, meaning of or -'s in English), 之 (zhi, meaning almost the same as 的), or 地 (de, used to modify an action, i.e. a verb) is needed to indicate the function of the modifier. For example, riot + helmets, security + guards, barefoot + boy, down the street + run, about the name change of the school (the actual order in Chinese is: about + school + change + name) + meeting.

\section{CONCLUSION}

Human language evolved for, from and with the expression of human communotion. The source, the basic structures, the mechanism and the generation of human communotion are universal to mankind. The alleged typology of human language is in reality the conventional verbalization in the realization of the expression of human communotion in different cultural communities.

Two main underpinning forces can be identified in the evolution of human language: the pressure of the expression of the communotion and the sociocultural convention in the realization of the verbalization of the expression of the commnotion. Admittedly, it had been a long history before animals developed conscious intention of communotion, but unquestionably, human language evolution did not begin until our simian ancestors reached that stage of conscious intention of communication, which emerged from the internal need of the something in Engels' discussion that impelled the men in the making to communicate to each other.

It is reasonable and natural for investigators of human language to approach it by severing a branch of research domain from the entire tree of human language phenomenon as their target of language study. In the first half of the $20^{\text {th }}$ century, we have Saussure's semiotic theory on 'langue' rather than 'parole', and, in the latter half of the century, we have Chomsky's transformational generative grammar centering on the human faculty of recursion of the hierarchical structures of human language and especially the production of grammar of the 
ideal English speaker, and Halliday's systemic functional grammar focusing on English grammar in use as a tool of social communication.

The development of all branches of language study has pointed to the ultimate issue in the study of human language: unraveling the myth of human language evolution. Verbal language originated from non-verbal communotion. The ultimate resolution of the myth of human language lies in the elucidation of the transformation or transduction from the non-verbal communotion to the verbal language. Although the concept of human communotion has never been under the spotlight of linguistics and language-related research areas, it has been relentlessly functioning there in the dominant theories of language studies, underpinning and supporting their discussions. For the first time in history, human communotion steps into the spotlight of attention in the discussion of issues in linguistics.

Saussure [11] designated the acquired language in the native speaker's mind as the object of language study, but when he analyzed the symbolic and semiotic nature of language, he had to resort to the mental concepts that the speaker developed through experiencing the outside world (e.g. a tree or a horse). The merger between the concept of a tree (the signified) and the form of sound image (signifier) in the speaker's mind is completed first by developing the concept through the lived and experienced world of the Dasein and then by associating the sound image with the concept in the mental manipulation of the content of communotion under the pressure of social communication. Chomsky [21-23] concerns himself exclusively with the grammar of human language (especially English), but the hierarchical structures could never be understood, not even be identified, without the semantic meaning of the structures, and the relations between sentences as the active-passive relation, discussed in his seminal work [22: Preface] could never be rendered possible without the mental manipulation of the content of communotion. His well-known example of colorless green ideas sleep furiously [22: 15] is in reality the speaker's syntactic knowledge gained through repetition of the English syntactic pattern of the communotion. Where Chomsky claims that "the notion 'grammatical' cannot be identified with 'meaningful' or 'significant' in any semantic sense," every word in the sentence and every suffix are actually meaningful. The reason why a native speaker can read this meaningless group of words with normal intonation is that every word in the group is located in its normal place of a normal English sentence. This is the result of the repetition of language behavior about conventional rules such as those of chess and language. These rules have been conventionally transformed and generated from the deep structure of non-verbal communotion. The failure of Chomsky's interpretation of his theories regarding the deep structure resulted from his failure of the recognition of human communotion. Throughout his whole life, Halliday endeavored to elucidate the relationships between human experience, language meaning, language structure and language function against the background of human social communication. Halliday [24] emphasized the dual function of the language as "action" and "reflection." Language is first the speaker's reflection of his lived and experienced world and then his action of languaging to express his communotion using the symbolic vocal sounds that were used to reflect the meaning from the experienced world. Although he identified the three levels of systems: the system of the lived and experienced world, the system of the culture or situation and the system of language, unfortunately he failed to formulate the theory of human communotion, which is the crucial point that can coordinate and align the languaging process across the three levels of reflection, action and language. If human language is viewed as a system, this system is the reflection of the system of the lived and experienced world of the Dasein; if it is considered to be functional, its function must have been concealed in the first action of human communication. The theory of communotion has elucidated the working, shaping and evolution of human language across the three systems. 
Like a genome in biology and genetics, human communotion is the genome of human language. Unlike a genome in biology and genetics, communotion is determined both by the nature of humans and by the culture that humans created. Human culture is the by-product in the process of human pursuit of the materialization of various intentions and desires through invention and creation of various tools. As a human tool, human language has never been the end of pursuit, but a means for the pursuit of the expression of the communotion under the pressure of communication. The refinement, perfection and development of human particular languages are all targeted towards better service of the expression of the communotion.

This article had demonstrated how the phenotypic syntactic structures of the Chinese language has been shaped and developed from the genomic information of the communotion and cultural convention of the Chinese community. I have placed human communotion at the center of human communication, with the lived and experienced world of the Dasein on one side and language created, on the other side, by merging the meaning from lived and experienced world (becoming the semantic meaning of the language) and the vocal sounds produced from the speech organs (becoming the form of the language). The hierarchical syntactic structures of human language are derived from the hierarchical structures of the elements of the communotion.

This article has justified the legitimacy of the Chinese language as one possible branch of human language and provided an analytical frame of parameters for the syntactic study of the Chinese language, not by referring to the Western frame, but by juxtaposing it with English under the same theory of human communotion. The inquiry into the Chinese language from the perspective of the evolution of the communotion also opens up new avenues for future research on the evolution of human language, the typology of human languages, syntax, pragmatics, language production, language acquisition, second language acquisition, and other language related areas.

\section{References}

1. X. Shen. The problem awareness, relational thinking and language consciousness in Chinese cultural linguistics. The Northern Forum, 2017, 261(1): 18-21.

2. J. Ma. Ma's Grammar. Beijing: Commercial Press, 1983.

3. M. Hu \& S. Jing. On flowing sentences. Language Teaching and Research, 1989, (4): 42-54.

4. J. Shen. On minor sentences and flowing sentences in Chinese: In commemoration of the $120^{\text {th }}$ birthday of Yuen Ren Chao. Studies of the Chinese Language, 2012, 350(4): 403-415.

5. M. Heidegger. Being and Time. Translated by J. Macquarrie and E. Robinson. Oxford: Basil Blackwell, 1962. (First published in 1927).

6. F. Engels. The part played by labour in the transition from ape to man, 1876. http://www.marxists.org/archive/marx/works/1876/part-played-labour/ (accessed May the 19th 2018 ).

7. L. S. Vygotsky. Thought and Language (newly revised and edited by Alex Kozulin). Cambridge, MA: Massachusetts Institute of Technology Press, 1986.

8. L. Bloomfield. Language. London: Goerge Allen \& Unwin Ltd, 1935.

9. T. Scott-Phillips. Speaking Our Minds: Why Human Communication is Different, and How Language Evolved to Make it Special. Basingstoke. Hampshire: Palgrave Macmillan, 2015.

10. E. Sapir. Language. An Introduction to the Study of Speech. NY: Harcourt, Brace \& World, 1949.

11. F. de .Saussure. Course in General Linguistics. Edited by Charles Bally and Albert Sechehaye with the collaboration of Albert Riedlinger (Roy Harris, Trans.), London: Gerald Duckworth \& Co. Ltd, 1983. (Original work published 1916.) 
12. C. Hoerl. Episodic memory and theory of mind: A connection reconsidered. Mind \& Language, 2018, 33: 148160. Doi: 10.1111/mila.12170.

13. M. A. Wheeler, D. T. Stuss \& E. Tulving. Toward a theory of episodic memory: The frontal lobes and autonoetic consciousness. Psychological Bulletin, 1997, 121(3): 331-354.

14. J. Russell. Episodic memory as re-experiential memory: Kantian, developmental, and neuroscientific currents. Review of Philosophy and Psychology, 2014, 5(3): 391-411. Doi: 10.1007/s13164-014-0194-3.

15. J. B. Mahr \& G. Csibra. Why do we remember? The communicative function of episodic memory. Behavioral and Brain Science, 2017, 41: 1-63. Doi: 10.1017/S0140525X17000012.

16. M. Soteriou. The epistemological role of episodic recollection. Philosophy and Phenomenological Research, 2008, 77(2): 472-492.

17. M. A. K. Halliday \& C. M. I. M. Matthiessen. Construing Experience through Meaning: A Language-based Approach to Cognition. London: Cassell, 1999.

18. Y. R. Chao. How Chinese logic operates. Anthropological Linguistics, 1959.1 (1): 1-8. (Operational Models in Synchronic Linguistics: A Symposium Presented at the 1958 Meetings of the American Anthropological Association.)

19. Y. Mo. Red Sorghum. Translated from the Chinese by Howard Goldblatt. London: Arrow Books, the Random House Group Limited, 2003. (First published in the United Kingdom in 1993 by William Heinemann Ltd.)

20. D. Zhu. Questions and Answers on Grammar. Beijing: The Commercial Press, 1985.

21. N. Chomsky. Aspects of the Theory of Syntax. Cambridge: MIT Press, 1965.

22. N. Chomsky. Syntactic Structures (Second Edition with an Introduction by David W. Lightfoot). Berlin, New York: Mouton de Gruyter, 2002. (Original work published in 1957.)

23. N. Chomsky. Language architecture and its import for evolution. Neuroscience and Biobehavioral Reviews, 2017, 81: 295-300.

24. M. A. K. Halliday. Systemic background. In J. D. Benson, \& W. S. Greaves (Eds.), Systemic Perspectives on Discourse, 1: 1-15. Norwood, NJ: Ablex, 1985. 